\title{
REFORMA POLÍTICA EN LA ORGANIZACIÓN DEL CONGRESO DE LA REPÚBLICA: ¿ES NECESARIO RESTITUIR EL SISTEMA BICAMERAL EN EL CONGRESO PERUANO?
}

\section{POLITICAL REFORM IN THE ORGANIZATION OF THE CONGRESS OF THE REPUBLIC: IS IT NECESSARY TO RESTITUTE THE BICAMERAL SYSTEM IN THE PERUVIAN CONGRESS?}

\author{
Dr. Dr. H.c. Ernesto Álvarez Miranda \\ Decano de la Facultad de Derecho de la Universidad de San Martín de Porres \\ ealvarezm@usmp.pe \\ Perú \\ Abg. Rosemary Ugaz Marquina \\ Abogada USMP \\ rosemary_ugaz@usmp.pe \\ Perú
}

\section{SUMARIO}

- Introducción

- Rol del Congreso de la República en el sistema constitucional peruano

- Democracia representativa en el sistema de representación unicameral y bicameral

- Elementos de análisis para el retorno a la bicameralidad.

- Conclusiones

\section{RESUMEN}

En el desarrollo del presente artículo, se analiza la posición institucional del Congreso de la República en el sistema constitucional peruano. Para ello, se explica en qué consiste el principio de democracia representativa y sus características principales en los sistemas de representación "unicameral" y "bicameral" del Congreso de la República. Posteriormente, se analizan los proyectos de ley que proponen restituir el sistema bicameral en el Congreso actual y se exponen los posibles efectos jurídicos y políticos que podría causar su retorno en la política peruana actual.

\footnotetext{
ABSTRACT

In the development of this article, the institutional position of the Congress of the
}

Republic in the Peruvian constitutional system is analyzed. To do this, it explains what the principle of representative democracy and its main characteristics in the "unicameral" and "bicameral" representation systems of the Congress of the Republic. Subsequently, the bills that propose to restore the bicameral system in the current Congress are analyzed and the possible legal and political effects that could cause its return in the current Peruvian politics are exposed.

\section{PALABRAS CLAVES}

Congreso de la República, bicameralidad, Democracia representativa, reforma constitucional.

\section{KEYWORDS}

Congress of the Republic, bicameral, Representative democracy, constitutional reform.

\section{INTRODUCCIÓN}

EL principio democrático contenido en el artículo $45^{\circ}$ de la Constitución, según el cual el poder del Estado emana del pueblo, implica que la titularidad de los cargos públicos sólo puede ser legítima si su origen, de manera mediata o inmediata, se funda en un acto concreto 
que expresa la voluntad popular, y siempre que su ejercicio se haga con las limitaciones y responsabilidades que la Constitución y las leyes establecen.

El Congreso de la República es el poder constituido por naturaleza de garantizar que la democracia representativa se concrete, siendo su principal herramienta su forma de organización.

Así de las cosas, nuestro último proceso constituyente, reformó la organización del referido Poder del Estado, decisión respaldada ante la crisis política existente a inicios de los noventa y la ineficiencia de la labor legislativa. Es así como, nuestra constitución actual, en su artículo $90^{\circ}$ identifica la composición del Congreso de la República como una cámara única.

Por ello, consideramos que el debate constitucional, respecto al retorno a la bicameralidad del Congreso de la República, es realmente importante para un análisis serio respecto a la reforma política del Estado.

En el desarrollo de las siguientes páginas, identificaremos el rol que cumple el Congreso de la Republica en optimizar la democracia representativa, así como la justificación constitucional del regreso a la composición bicameral del Congreso de la República.

\section{ROL DEL CONGRESO DE LA REPÚBLICA EN EL SISTEMA CONSTITUCIONAL PERUANO}

Desde nuestra Constitución, el Congreso de la República se identifica como la institución constitucional que cumple funciones de representación, legislación y control (artículo $102^{\circ}$ de la Constitución), es decir, un poder constituido encargado de ser el "órgano representativo de la Nación" (artículo $2^{\circ}$ de su Reglamento Normativo)

Es decir, se rige bajo el principio de democracia representativa, la cual desde el artículo $43^{\circ}$ de la Constitución forma parte de su propia esencia como poder del Estado, cuya función primordial es hacer realidad dicho principio constitucional en nuestra comunidad política, el cual se organiza en una única cámara de representación de acuerdo al artículo $90^{\circ}$ de la Constitución.
Para mayor desarrollo, el Congreso de la República se refleja en nuestra Constitución, desde la composición del artículo $45^{\circ}$ de nuestra Constitución, que establece que "el poder del Estado emana del pueblo", y quienes lo ejercen "lo hacen con las limitaciones y responsabilidades que la Constitución y las leyes establecen".

Lo anterior, va de la mano con el artículo $43^{\circ}$ de la misma Carta nos hace mención que el Perú es una república "democrática", así como social, independiente y soberana; y que su gobierno es, además de unitario y descentralizado, "representativo", el cual se organiza según el principio de separación de poderes.

En ese sentido, el Congreso de la República es un órgano deliberativo por naturaleza, es así que el Tribunal Constitucional Peruano, en su jurisprudencia ha desarrolla que:

" $[u]$ no de los espacios idóneos y predominantes para materializar dicho proceso de deliberación es el Congreso. En efecto, este es un auténtico órgano deliberante" (Tribunal Constitucional, 2017).

Por ello, es necesario que la práctica deliberativa del Congreso peruano venga determinada por la finalidad de

"enriquecer el debate no solo en cuanto a lo referido a la información que se pueda brindar, sino también en cuanto a perspectivas y enfoques. $Y$ es que es la calidad del proceso deliberativo la que legitima la función legislativa, de ahí que se afirme que una norma jurídica se legitima como resultado de la deliberación general, y no de la voluntad general", (Tribunal Constitucional, 2017).

Coincidimos en que la misión central del parlamento es de carácter deliberativo. Es decir, presentar los diferentes puntos de vista e intereses de la sociedad con la finalidad de llegar a acuerdos políticos.

Actualmente, existe una falta de representatividad y probablemente sea adecuado repotenciar los mecanismos que la Constitución provee para que el Parlamento "refuerce su vital rol institucional, cual es el de ser un verdadero canal y puente entre las aspiraciones ciudadanas y las políticas gubernamentales". (Tribunal Constitucional, 2006) 
Political reform in the organization of the congress of the republic: is it necessary to restitute the bicameral system in the peruvian congress?

En el desarrollo de líneas posteriores, identificaremos las principales características de la democracia representa y su relación con la organización del Congreso de la República.

\section{DEMOCRACIA REPRESENTATIVA EN EL SISTEMA DE REPRESENTACIÓN UNICAMERAL Y BICAMERAL EN EL CONGRESO DE LA REPÚBLICA}

Para el Tribunal Constitucional Peruano, la democracia representativa, es entendida como aquella forma de autogobierno en la que "existe la conjugación armónica del principio político de soberanía popular con un cauce racional de deliberación que permita atender las distintas necesidades de la población" (Tribunal Constitucional, 2005).

Por ello, la relación entre Constitución, Parlamento y democracia representativa, resulta consustancial a nuestro sistema político, conforme a la definición del Poder Legislativo como aquel cuya autoridad ha sido delegada por el Pueblo como fuente originaria del poder.

Por tanto, la legitimidad democrática del Congreso depende de su capacidad para generar esa racionalidad deliberativa en el ejercicio de sus funcione.

Podemos partir de la descripción del sistema unicameral de representación en el Congreso de la República, establecido en el artículo $90^{\circ}$ de la Constitución parte de un modelo propio del período de reformas que se inició en 1992 , y qué a decir de la doctrina, se caracterizó "por menores niveles de representación y de deliberación, a favor del menor costo de la institución parlamentaria y la toma más rápida de decisiones" Delgado-Guembes, (2011).

Dicho sistema, sin embargo, ha sido objeto de variadas críticas, las cuales afirman el fracaso de dicho sistema "expresado en su absoluta sumisión al poder presidencial, la renuncia a su función de control y la falta de calidad de la producción legislativa, condicionada exclusivamente por las necesidades y conveniencias del gobierno" (Blancas Bustamante, 2001,p. 45)

Aun cuando en tradición constitucional, bicameralismo ha sido la regla general, también es verdad que durante el siglo XX dicho sistema fue objeto de severos ataques que reprochaban su carácter elitista o, en otros casos, en su excesiva politización.

Así, cambia nuestro paradigma constitucional de inicios de los años 90's propuso establecer el debate de si las reformas serían vitales para lograr que nuestro Congreso de la República sea más representativo y que, por ende, permita abrir este espacio público a los intereses de todos los representados. Desde luego, dicha tarea seguramente "habrá de partir del reforzamiento de los partidos políticos, que son los que ofrecen al electorado los candidatos idóneos para representarlos" (Tribunal Constitucional, 2006); pero, centrando por ahora nuestra atención en el Poder Legislativo como institución, el fin que de éste ha de esperarse no puede ser otro que el de convertirse en un "auténtico foro deliberativo, vale decir, un espacio donde el intercambio de ideas a favor y en contra de una posición determinada permite que la decisión finalmente adoptada represente los intereses de todos" (Nino, C, 1997, p. 161), y genere como resultado una verdadera Waldron (2016) "legislación representativa".

Así de las cosas, el profesor José Miguel Rojas Bernal (2017), en su estudio al respecto, propuso que de los diversos diagnósticos que han sido efectuados sobre el sistema unicameral en el Perú, y su impacto sobre el nivel de representatividad del Congreso peruano, es posible mencionar cuando menos las siguientes:

1. Problemas en la selección de los temas de la agenda del Congreso peruano: como ha referido la doctrina, el unicameralismo ha tenido como uno de sus resultados negativos, el que la agenda de las sesiones se fije por un "criterio de coyuntura", por "reciprocidad entre las distintas agrupaciones parlamentarias", o también por la "capacidad de agencia e influencia del gobierno". En ese contexto, lo que finalmente se debate en las comisiones o en el Pleno no se selecciona en base a la importancia o prioridad de los temas, "sino en función de las distintas agendas de los actores interesados en atender demandas populares de las localidades o de potenciales electores"

2. Uso excesivo de la exoneración de la segunda votación: se ha señalado así que la regla de la obligatoriedad de la segunda 
votación "en la práctica se exonera en más del $90 \%$ de los casos, con lo cual el proceso de recalentamiento se intensifica privando a los representantes de espacios adicionales de prevención, prudencia y reflexión". De este modo, por ejemplo, en el quinquenio parlamentario 2006-2011, fueron exonerados de segunda votación, por el Pleno del Congreso de la República, "572 dictámenes de 626 que requerían ese trámite. Es decir, el $91.37 \%$ de los dictámenes que requerían de segunda votación fue exonerado. Sólo fueron votados en segunda votación 54 dictámenes" (Proyecto de Ley N²341/2012-CR).

3. Escaso nivel de debate de los proyectos de ley a nivel de las comisiones ordinarias: en efecto, a raíz de las últimas modificaciones que han tenido por objeto reducir el número de los debates en el Pleno y trasladar esa competencia deliberativa a las comisiones, se ha generado un "sobredimensionamiento" del papel de las comisiones ordinarias; pese a lo cual, existe evidencia de que los congresistas que integran dichas comisiones no suelen investigar, estudiar ni preparar su participación en el debate (Reflexión democrática, 2012) , lo que genera que el trabajo a nivel de comisiones genere una "puerta abierta para el procesamiento laxo de propuestas normativas, conforme al cual se transfiere a las Comisiones alternativas legislativas impropias o inconvenientes" (Delgado Guembres, 2014). Por ello, se afirma con razón que este procedimiento de "desconcentración" del Pleno y "sobredimensionamiento" de las comisiones no ha permitido asegurar "la aprobación de normas socialmente convenientes o necesarias, sino cumplir con la formalidad de las reglas que permiten que la mayoría convierta en ley lo que quiera que haya sido incluido en la agenda de la sesión, sin plan alguno ni objetivos nacionales o institucionales" Idem. Ese escenario no cambia si se tiene en cuenta el escaso número de consultas que son realizadas por los congresistas a la sociedad civil sobre los proyectos de ley que se proponen y debaten en el Congreso (Asociación civil transparencia, 2017).

4. Finalmente, ello parece tener como correlato el considerable número de autógrafas que son observadas por el Ejecutivo, en los últimos años.
5. Ineficienciaenlaeleccióndealtos funcionarios del Estado: según nuestra Constitución, el Congreso de la República tiene la función de designar y/o ratificar a los miembros de ciertos organismos constitucionalmente autónomos, tales el Contralor General de la República, el Defensor del Pueblo, los miembros del Tribunal Constitucional, el Directorio del Banco Central de Reserva y el Superintendente de Banca y Seguros; y asimismo, le corresponde removerlos en los casos previstos en la Constitución (artículo $6^{\circ}$ del Reglamento Normativo del Congreso). Sin embargo, como es de conocimiento general, el Congreso de la República no ha venido cumpliendo dicha función con la debida diligencia, no sólo en lo que se refiere a la oportunidad de los nombramientos, sino también al perfil de los candidatos que son finalmente elegidos, con las consecuencias negativas que todo esto supone para la buena marcha de aquellas instituciones.

Es por lo anteriormente señalado que, el sistema unicameral, junto a otros factores de nuestro sistema político, no se proponen como mecanismos adecuados para concretar el principio de democracia representativa en sede parlamentaria.

Por ello, es importante poner en discusión para la reforma política en el país, el cambio constitucional de organización del Congreso de la República, es decir, si es viable la alternativa bicameral y de ser así, cómo ésta podría ser implementada en nuestro país.

\section{ELEMENTOS DE ANÁLISIS PARA EL RETORNO A LA BICAMERALIDAD}

Para definir el sistema bicameral de organización del parlamento, debe tener en cuenta su origen que "es fruto del compromiso entre fuerzas políticas, expresa la heterogeneidad del cuerpo social y sirve para el compromiso y la moderación" (Sánchez de Dios, M., 2004).

También se describe a este mismo sistema como el capaz de enfrentar "disfunciones parlamentarias como los problemas de la tiranía de la mayoría, de la tiranía de las minorías, o el del voto circular", además de "entrenar a la clase política antes de pasar al primer plano, o bien para recoger su experiencia en la fase de retiro" (op. cit. 2004). 
Por ello, las virtudes de este sistema han sido bien justificadas a través de la denominada "dualidad de niveles de representación" que pretendió garantizar el liberalismo; es decir, "[a] la voluntad considerada superficial y contingente surgida del voto popular directo, se opuso la necesidad de una representación de la nación más profunda y menos propensa a las variaciones de opinión" (Garrido López, 2016, 65).

Desde la experiencia comparada, el sistema bicameral también ha recibido críticas, cuando éste no resulta funcional al fin constitucional que pretende optimizar. Así, por ejemplo, cierto sector de la doctrina afirma que, en los Estados federales, la segunda Cámara no funciona como Cámara territorial "sino que reproduce la misma dinámica partidista de la primera Cámara. En la práctica, la supuesta representación especial carece de especialidad territorial y deviene representación política mediada por partidos" (Garrido López \& Saenz, 2014).

Por el contrario, para esta corriente doctrinaria, la incorporación de los "intereses políticos territoriales" no requiere crear una segunda Cámara, sino que ello depende "del sistema electoral y de los partidos que operan en los dos niveles territoriales" idem. Por tanto, se llega a afirmar, que desde una perspectiva comparada las Segundas Cámaras federales no son verdaderos cauces de una representatividad alternativa a la ofrecida por el sistema de partidos, y en ese sentido su declive se expresa en que "han derivado en instituciones irrelevantes (caso de Canadá, Austria y Bélgica), se han convertido en cámaras representativas del mismo pueblo ya representado, y de forma mucho más representativa, en la Cámara baja (caso de EE.UU. y Suiza), o son utilizadas por la oposición como instrumento de bloqueo contramayoritario frente a la acción del Gobierno federal (caso de Australia)".

Ello explicaría el retroceso del bicameralismo en varios sistemas políticos de Estados federales y sin estructura federal (Garrido López, 2014).

Si bien al unicameralismo se le reprocha el constituir una forma de acelerar los procesos parlamentarios, que "tiende en efecto a empeorar la capacidad productiva del régimen representativo, porque se minimiza la discusión de las objeciones, se carece de un punto de inflexión cuando hay falta de concordia entre el gobierno y el parlamento; lo cierto es que el "argumento de la mayor reflexión" como ventaja comparativa del sistema bicameral sería falaz, pues la Cámara Alta "está reclutada no menos políticamente que la Cámara Baja”.

En principio se afirma con razón, que la mayor reflexión no sería consecuencia del número de cámaras, sino de la calidad de los representantes.

Dicho en otras palabras: la mejor asamblea no es la que tiene el diseño institucional perfecto, sea de una o de dos Cámaras, sino la integrada por representantes probos, capaces y dedicados a la República".

Sin embargo, no puede dejarse de lado, que la principal ventaja el dotar al Congreso de una segunda cámara revisora (o como elemento de control): dejar de lado que la toma de decisiones en el ámbito parlamentario siempre obedecerá a razones políticas, motivo por el cual por más que se pretenda considerar al senado como la 'cámara reflexiva', ésta será siempre tan 'política' como la 'cámara joven'. Las decisiones del senado, tal como ocurre en la cámara de diputados, serán "el resultado de la correlación de fuerzas, de las alianzas o acuerdos políticos a los que puedan arribar las bancadas, más que a la reflexión, al análisis o la revisión serena de los proyectos aprobados en primera instancia en la primera cámara" (Salcedo Cuadros, C, 2015, 67)

Por ello, una reforma constitucional en la organización del Congreso Peruano, no solo será ventajosa porque

“se asigna al Senado un marco de
responsabilidades basado en un conjunto
distinto de temas respecto del cual tiene
competencias excluyentes respecto de
la Cámara de Diputados, y además
es integrado o elegido según criterios
disímiles a aquellos según los cuales
se elige a la Cámara de Diputados, es
predecible que la mayor diversidad y
el carácter bicameral (...) mejorará en
efecto la capacidad de la institución
parlamentaria para agregar valor público
en el proceso de transformación de la
demanda en resultados corporativos”
(Delgado Guembes, 2014).

" se asigna al Senado un marco de responsabilidades basado en un conjunto distinto de temas respecto del cual tiene competencias excluyentes respecto de la Cámara de Diputados, y además es integrado o elegido según criterios disímiles a aquellos según los cuales se elige a la Cámara de Diputados, es predecible que la mayor diversidad y el carácter bicameral (...) mejorará en efecto la capacidad de la institución entaria para agregar valor publico demanda en resultados corporativos" 
Sino porque, desde nuestro aporte, consideramos el retorno a la bicameralidad se justifica en la medida que cumple con garantizar las dos funciones naturales del Congreso de la República: la deliberativa y de representación.

Para la garantía de esta última, es necesaria una representación de un primer tipo, es decir, directa, de la comunidad con su líder. Con ello, los electores de un distrito electoral determinaran a su representante, con la finalidad de intermediar tendencias e intereses de manera directa, lo cual conlleva el reclutamiento de líderes locales, los cuales conformarían la cámara de diputados.

Lo anterior debe complementarse con la representación de segundo tipo, la del Bien Común, es decir, la de los intereses nacionales, a cargo precisamente de representantes elegidos en distrito nacional único, que deben su elección y deben tratar de reelegirse no atendiendo a determinada circunscripción o determinado grupo social localizado de interés, sino a tendencias e intereses de carácter nacional, grandes corrientes de opinión que se expresan por encima de la territorialidad de provincias y regiones, los cuales conformarían la cámara de senadores.

Teniendo en cuenta estas premisas, observaremos si los proyectos de ley de reforma del sistema de organización del Congreso de la República cumplen con las observaciones planeadas.

Al respecto podemos observar que, en el caso del Proyecto de Ley 1678/2016-CR, se propone que la Cámara de Senadores sea elegida por las Regiones (dos por cada una de ellas), mientras que la Cámara de Diputados, lo sea por distrito electoral, en número proporcional a sus electores, conforme a Ley. Por su parte, el Proyecto de Ley 1740/2017$\mathrm{CR}$, plantea que mientras el Senado se elige por distrito electoral único (garantizando así una suerte de "representación nacional"), la Cámara de Diputados sería elegida por distrito electoral múltiple (favoreciendo con ello una "representatividad territorial").

Es decir, en el primer proyecto pretende también impulsar la descentralización mediante la elección de los senadores desde un punto de vista regional, mientras que el segundo proyecto, propone un retorno semejante a la organización de la Constitución de 1979.
Desde un punto de vista general, ambos proyectos de ley apuntan a un modelo de bicameralismo "desigual" o "imperfecto" en el que las dos cámaras de distinta composición, poseen distintas funciones y un similar poder de veto.

Para ambos proyectos, el perfil de los miembros de cada cámara asegura una mirada distinta sobre las necesidades de la representación nacional.

Se exige que los senadores y diputados sean peruanos de nacimiento y gocen de derecho de sufragio. Sin embargo, también establecen que, para acceder al Senado, el ciudadano debe haber cumplido 35 años de edad (a diferencia de los 25 años de edad que se exige para ser diputado), y en el caso específico del Proyecto de Ley N. $1678 / 2016-C R$, agrega que el senador debe ser profesional o haber sido diputado por 2 períodos.

Las referidas limitaciones, si podrían permitirse en nuestro ordenamiento jurídico al ser un ejercicio legítimo de la restricción al derecho a ser elegido, en tanto se trata de restricciones que no sólo están permitidas por la Constitución y la Convención Americana, sino que además devienen en razonables y proporcionales al fin que pretenden optimizar, cual es el principio de democracia representativa, lo cual pasa por garantizar un adecuado perfil de quienes han de integrar una cámara cuya función será revisar con mayor criterio técnico y especializado lo que ha sido aprobado en una primera instancia.

Por tanto, se encuentra justificada las características específicas que pueden diferenciar a un diputado y un senador, así como la diferencia en sus funciones, pues tal como indica el profesor Sartori si las dos cámaras son muy parecidas, se facilita la gobernabilidad, pero no sirve al propósito de control o balance; pero si son diferentes "aseguran un mayor control, pero también prometen conflicto y estancamientos" (Sartori, 1999, p.201).

Por esa razón, el autor sostiene que, a mayor desigualdad de poder entre las cámaras, debe favorecerse una mayor diferencia en la composición entre ellas, y a la inversa: "cuanto más fuerte sea el bicameralismo, tanto más debemos buscar su semejanza" (Sartori, 1999, p.203). 
Teniendo en cuenta las consideraciones planteadas, observamos que el proyecto Ley 1740/2017-CR, se encontraría más acorde a la visión de bicameralismo que planteamos, pues por un lado cámara de diputados tendría una presentación directa, al tener en cuenta la elección de líderes comunitarios de acuerdo a cada circunscripción, mientras que la elección del Senado se elige por distrito electoral único, con requisitos específicos que permitiría darle una connotación nacional a la representación de intereses y reflexión que dotarían de calidad a la producción normativa del Congreso de la República.

Finalmente, consideramos necesario la reforma de la organización del Congreso de la República y el retorno a la bicameralidad debe ser aprobado, esta premisa no vulneraría la fórmula política la Constitución.

Si bien, el sistema unicameral instaurado en la Constitución de 1993 no constituye un elemento "definidor" o "esencial" de nuestra forma política democrática, social, independiente y soberana (artículo $43^{\circ}$ ): más bien, nuestra constitución histórica, ha sido el sistema bicameral el que ha formado parte de nuestra tradición constitucional, y el constituyente tampoco ha manifestado que el diseño actual del Congreso peruano sea una cláusula irreformable.

Por su parte, el Tribunal Constitucional tampoco ha incluido al sistema unicameral del Congreso peruano (artículo $90^{\circ}$ ) dentro de aquellas cláusulas que brindan identidad a nuestro texto constitucional y son límites materiales al poder de reforma (Tribunal Constitucional, 2002).

Por tanto, podemos afirmar que debemos que la reforma constitucional dirigida a restaurar el sistema bicameral resulta no sólo constitucionalmente viable, sino plenamente exigible a partir del principio de democracia representativa que nuestra Constitución reconoce, y cuya maximización en sede del Congreso peruano resulta de primer orden.

\section{CONCLUSIONES}

El Congreso de la República es un órgano deliberativo por naturaleza, encargado de representar, legislar y fiscalizar de acuerdo al mandato constitucional. Su rol constitucional es ser el garante de la democracia representativa tal como señala el artículo 43 de la Constitución Política, de tal manera que se convierte en el foro legislativo al ser un espacio público idóneo para el proceso deliberativo.

Nuestra Constitución actual, recoge la organización unicameral de organización del Congreso de la República, pero como se vio en líneas anteriores, el modelo no contribuye en optimizar la democracia representativa.

Proponemos que las propuestas de retorno a la bicameralidad del Congreso de la República, deben de tener en cuenta que, la forma de elección de la cámara de diputados, los electores de un distrito electoral determinaran a su representante, con la finalidad de intermediar tendencias e intereses de manera directa, lo cual conlleva el reclutamiento de líderes locales, mientras que, en la cámara de senadores, la forma de elección debe ser por distrito electoral único, ya que su elección no atiende a determinada circunscripción o determinado grupo social localizado de interés, sino a tendencias e intereses de carácter nacional.

Finalmente, la propuesta de retorno a la organización bicameral del Congreso de la República, constituye una opción necesaria para elevar los niveles de representatividad y eficiencia del Congreso peruano; sin embargo, para lograr ese cometido, es necesario que la segunda cámara no se limite a ser una instancia "revisora" de lo aprobado por la cámara baja; sino que ambas cámaras cuenten con una composición distinta y con funciones claramente distinguibles, que logren concretar el principio de democracia representativa que funda la razón de ser del Congreso de la República.

\section{FUENTES DE INFORMACIÓN}

\section{Fuentes bibliográficas}

Nino, Carlos Santiago (1997) "La constitución de la democracia deliberativa." Barcelona: Gedisa

Sartori, G. (1999) Ingeniería constitucional comparada. Una investigación de estructuras, incentivos y resultados, traducción de Roberto Reyes, $3^{a}$ reimpresión. México: Fondo de Cultura Económica. 
Blancas Bustamante, C. (2001): "La reforma constitucional del Congreso", en Derecho \& Sociedad, N. ${ }^{\circ} 17$.

Sánchez de Dios, M (2004) “¿Es necesario el Senado en los sistemas federales?", en Revista de las Cortes Generales, N. ${ }^{\circ} 63$.

Reflexión democrática (2012) "Balance y lecciones aprendidas durante el período parlamentario 2006-2011". Estudio retrospectivo con la participación de congresistas reelectos, mayo de 2012, Lima.

Delgado-Guembes, C (2012) "Manual del Parlamento: introducción al estudio el Congreso Peruano." Lima: Fondo editorial del Congreso de la República

Garrido López, C y Eva Saénz (2014): "Razones para plantear la supresión del Senado", en Cuadernos Giménez Abad, N. $7^{\circ}$.

Salcedo Cuadro, C (2015) "Propuesta para una reforma integral del Parlamento peruano", en Cuadernos Parlamentarios. Revista especializada del Centro de Capacitación y Estudios Parlamentarios, Núm. 13, Lima.

García Jaramillo, L. (2015) Constitucionalismo deliberativo Estudio sobre el ideal deliberativo de la democracia y la dogmática constitucional del procedimiento parlamentario. México: UNAM

Delgado Guembes, C. (2015) Olvido constitucional y vacio representativo en el Perú. Lima: Fondo Editorial del Congreso del Perú, Lima,

Garrido López, C. (2016) "Pero... ¿puede ser el Senado una Cámara de representación territorial?", en Revista Española de Derecho Constitucional, N. ${ }^{\circ} 107$, mayo-agosto.

Waldron, Jeremy (2016) "Representative Lawmaking", en Political Political Theory. Essays on Institutions, Harvard University Press.
Rojas Bernal, José Miguel (2017) Informe al Congreso de la República respecto a los proyectos de ley que proponen el retorno a la Bicameralidad. Centro de Estudios de Derecho Constitucional- Derecho USMP

Asociación Civil Transparencia (2017) Observatorio de Desempeño Parlamentario 2017, Lima

Tribunal Constitucional, 2017. STC N. ${ }^{\circ}$ 00062017-PI/TC, fundamento 4.

Tribunal Constitucional, 2006. STC N. ${ }^{\circ} 0026-$ 2006-AI/TC, fundamento 18

Tribunal Constitucional, 2006. STC $\mathrm{N}^{\circ}$ 00262006-AI/TC, fundamento 8

Tribunal Constituciona, 2005. STC N. ${ }^{\circ} 0030-$ 2005-PI/TC, fundamento 9.

Tribunal Constitucional, 2003. STC N. ${ }^{\circ} 0014-$ 2003-AI/TC, fundamento 28.

Fuentes electrónicas

Exposición de Motivos del Proyecto de Ley N. ${ }^{\circ} 2342 / 2012-C R$, de fecha 12 de junio de 2013, que propone modificar el artículo $73^{\circ} \mathrm{del}$ Reglamento del Congreso de la República.

Delgado Guembes, C (2014) "Para examinar el retorno a la bicameralidad en el Perú", s/f, [disponible en: https://es.scribd.com/ document/213755966/CDG-Para-examinarel-retorno-a-la-bicameralidad-en-el-Peru-2014

Congreso de la República (2016) Indicadores de Gestión. Período Anual de Sesiones 2015-2016, Congreso de la República, disponible en: http://www.congreso.gob.pe/ Docs/DGP/GestionInformacionEstadistica/ files/indicadores/indicadores_de gesti\%C3\%B3n_15.7.2016.pdf 